\title{
Potential prognostic factors in progression- free survival for patients with cervical cancer
}

\author{
Hui-Hui Chen ${ }^{1 \dagger}$, Wei-Yu Meng ${ }^{2 \dagger}$, Run-Ze Li ${ }^{2+}$, Qing-Yi Wang ${ }^{1}$, Yu-Wei Wang ${ }^{2}$, Hu-Dan Pan ${ }^{2}$, Pei-Yu Yan ${ }^{2}$,
}

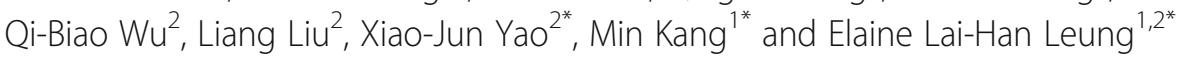

\begin{abstract}
Background: Cervical cancer continues to be one of the leading causes of cancer deaths among females in low and middle-income countries. In this study, we aimed to assess the independent prognostic value of clinical and potential prognostic factors in progression-free survival (PFS) in cervical cancer.

Methods: We conducted a retrospective study on 92 cervical cancer patients treated from 2017 to 2019 at the Zhuhai Hospital of Traditional Chinese and Western Medicine. Tumor characteristics, treatment options, progressionfree survival and follow-up information were collected. Kaplan-Meier method was used to assess the PFS.

Results: Results showed that the number of retrieved lymph nodes had a statistically significant effect on PFS of cervical cancer patients $(P=0.002)$. Kaplan-Meier survival curve analysis showed that cervical cancer patients with initial symptoms age $25-39$ had worse survival prognoses $(P=0.020)$. And the using of uterine manipulator in laparoscopic treatment showed a better prognosis $(P<0.001)$. A novel discovery of our study was to verify the prognostic values of retrieved lymph nodes count combining with FIGO staging system, which had never been investigated in cervical cancer before. According to the Kaplan-Meier survival curve analysis and receiver operating characteristic (ROC) curve analysis, significant improvements were found after the combination of retrieved lymph nodes count and FIGO stage in predicting PFS for cervical cancer patients ( $P<0.001, \mathrm{AUC}=0.826,95 \% \mathrm{Cl}: 0.689-0.962)$.

Conclusion: Number of retrieved lymph nodes, initial symptoms age, uterine manipulator, and retrieved lymph nodes count combining with FIGO staging system could be potential prognostic factors for cervical cancer patients.
\end{abstract}

Keywords: Cervical cancer, Progression-free survival, Retrieved lymph nodes count, FIGO staging

\footnotetext{
*Correspondence: xjyao@must.edu.mo; 53683127@qq.com;

Ihleung@must.edu.mo

${ }^{\dagger}$ Hui-Hui Chen, Wei-Yu Meng and Run-Ze Li contributed equally to this work.

${ }^{2}$ State Key Laboratory of Quality Research in Chinese Medicine/Macau

Institute for Applied Research in Medicine and Health, Macau University of

Science and Technology, Macau, People's Republic of China

'Zhuhai Hospital of Traditional Chinese and Western Medicine, Zhuhai City,

Guangdong, People's Republic of China
}

(c) The Author(s). 2021 Open Access This article is licensed under a Creative Commons Attribution 4.0 International License, which permits use, sharing, adaptation, distribution and reproduction in any medium or format, as long as you give appropriate credit to the original author(s) and the source, provide a link to the Creative Commons licence, and indicate if changes were made. The images or other third party material in this article are included in the article's Creative Commons licence, unless indicated otherwise in a credit line to the material. If material is not included in the article's Creative Commons licence and your intended use is not permitted by statutory regulation or exceeds the permitted use, you will need to obtain permission directly from the copyright holder. To view a copy of this licence, visit http://creativecommons.org/licenses/by/4.0/ The Creative Commons Public Domain Dedication waiver (http://creativecommons.org/publicdomain/zero/1.0/) applies to the data made available in this article, unless otherwise stated in a credit line to the data. 


\section{Background}

Globally, cervical cancer is the fourth of the most common cancers among females after breast, colorectal, and lung cancer [1]. In low and middle-income countries (LMICs), cervical cancer continues to be the second most prevalent cancers in morbidity and one of the leading causes of cancer deaths among females [2, 3]. It is a remarkable cause of mortality and morbidity among youthful and middle-aged women aged 20 to 39 years all over the world [4]. In January 2019, the National Central Cancer Registry of China (NCCRC) released its latest nationwide tumor statistics of population-based tumor registry data in 2015 gathered from 368 tumor registries in China. According to the number of female patients, cervical cancer ranks sixth in the incidence of malignant tumors in China. The overall crude incidence rate by Chinese standard population for cervical cancer was $16.56 / 100,000$. Nearly 111,000 new cervical cancer cases were diagnosed in 2015, which alone has around $18.7 \%$ of the total cervical cancer in the world [3]. Due to the lack of valid prevention and screening methods, it is worth noting that morbidity of cervical cancer is still increasing in less-developed countries in recent years $[5,6]$. Therefore, it appears to be an important step to find out potential prognostic factors in progression-free survival for patients with cervical cancer.

The prediction of clinical prognostic is the key factor in the therapeutic decision-making process. For cervical cancer, the therapeutic strategy generally depends on the clinical stage, which is established by the FIGO (International Federation of Gynecology and Obstetrics) staging system. The FIGO staging system exposes the extent and aggressiveness of cervical tumor, which is one of the known predictors for survivals. Nevertheless, as the FIGO staging was mainly based on clinical assessment of the anatomic extent, the error rate between the final histopathologic classification and the FIGO staging is about $25 \%$ in patients with early stages $[7,8]$. Hence, it is inevitable to enhance the FIGO staging system for more precise and practical prognostication of cervical cancer patients, optimizing the life quality of long-term survival and individualized treatment.

Lymphadenectomy is the standard constituent part of cervical cancer patients treatment $[9,10]$. Retrieved lymph nodes (RLN) count is considered as the way of objectively measure of the radicalness and thoroughness of the surgical procedure [11]. The range of lymphadenectomy is a matter of debate in the treatment of cervical cancer. Theoretically, lymphadenectomy could enhance the probability of detecting occult metastatic disease. In a study based on the single-institution review of cervical cancer patients, it has been reported that cervical cancer patients with more retrieved lymph nodes had better progression-free survival (PFS) [12].
And there is evidence investigated by other researchers proved that a more extensive lymphadenectomy ( $>40$ RLNs) improved survival of cervical cancer patients with tumors sized $>4 \mathrm{~cm}$ [9]. However, proof on some serious adverse events suggests that cervical cancer patients with lymphadenectomy are more likely to undergo surgeryrelated systemic morbidity [13], and a more thorough lymphadenectomy could lead to postoperative complications and damage the immune system, such as lymphedema of the legs [14, 15]. Consequently, determining appropriate surgical scope could be conducive to avoid postoperative complications and improved survival of cervical cancer patients.

Traditional clinical variables, including tumor size, parametrial involvement, and so on, play important roles in the patient prognosis and FIGO staging system [16]. Nevertheless, conventional pathological variables are not reliable enough for finding optimal treatment strategies and predicting clinical prognosis. In terms of progression-free and overall survival, patients with similar pathological characteristics and clinical tumor stage have different prognosis. On account of the difficulty of predicting the prognosis of cervical cancer patients, it is urgent to find more novel clinical and prognostic factors for cervical cancer. Many factors, such as lymph node metastasis, initial symptom age, and days of catheter removal may provide effective information for appropriate therapeutic options and patient prognosis. In this study, we aimed to assess the independent prognostic value of clinical and potential prognostic factors in progression-free survival (PFS) in cervical cancer.

\section{Methods}

\section{Study design and participants}

A total of 100 cervical cancer patients of the Zhuhai Hospital of Traditional Chinese and Western Medicine were followed-up in this study between January 2017 and October 2019. Our daily diagnosis and treatment were in strict accordance with the guideline of the 2017 and 2019 NCCN. Totally, we included 100 cervical cancer patients, in whom 93 patients were cervical cancer of stage I-II, 6 patients were stage III, and the other one patient was cervical cancer stage IVa. According to the guideline, these seven patients were recommended to receive radiotherapy and chemotherapy, and were told that surgical treatment was not recommended. However, these patients insisted and strongly demanded surgical treatment, thus they voluntarily signed up for the surgery and was included in the follow-up plan. The exclusion criteria: according to the guidelines of the $\mathrm{NCCN}$ and clinical cervical cancer criteria, patients with previous other tumors receiving chemotherapy as well as radiotherapy and with surgical contraindications that 
cannot receive surgery were excluded from this study. Among the 100 patients, 7 cases were diagnosed with precancerosis after surgery and one case with relapse were also excluded from following analysis. Finally, there was 92 patients included in the following analysis. Lymph node metastases were diagnosed by pathological examination after lymphadenectomy. This study was approved by the institutional ethics committee and informed consent was obtained from all cervical cancer patients. Follow-up duration was from the date of primary therapy to the date of death or last contact (October 2019).

\section{Surgical procedures and pathological review}

All the patients included in this study received surgical treatment for the first time. Among them, based on the NCCN guidelines and patients' own decision, 5 patients underwent laparoscopic extensive total hysterectomy and bilateral salpingectomy (3 of stage Ia1, 1 of stage IVa, and 1 of stage IIIb) and other 87 patients underwent laparoscopic radical hysterectomy, pelvic lymph node dissection and partial para-aortic lymph node dissection. Twenty-four patients were submitted to bilateral salpingo-oophorectomy (4 of stage Ia1, 14 of stage Ib1, 3 of stage Ib2, 1 of stage IIb1, 1 of stage IVa, and 1 of stage IIIa). The extent of para-aortic lymph node dissection (PALND) transformed from para-aortic lymph node sampling to thorough lymphadenectomy up to the level of the renal vein. Pelvic lymph node dissection (PLND) included dissection of the internal iliac, external iliac, common iliac, and obturator lymph nodes on both sides. Parametrial lymph nodes were removed along with the parametrium. Para-aortic nodes and pelvic lymph nodes were both included in the retrieved lymph nodes count. Surgical techniques will improve over time. At the time of 2017, NCCN guidelines recommend radical total hysterectomy plus bilateral pelvic lymphadenectomy as the preferred surgical option and sentinel lymph node biopsy as an alternative method. Nowadays, sentinel lymph node biopsy is the first choice of surgical method for the patients with tumor less than $2 \mathrm{~cm}$ according to the NCCN guidelines [17].

\section{Statistical analysis}

Kaplan-Meier method was used to assess the progression-free survival (PFS), and the log-rank test was used to analyze prognostic variables. Receiver operating characteristic (ROC) curve analysis was established to evaluate the prognostic performance of variables. $P$-value $<0.05$ was considered statistically significant. All statistical data were analyzed by using SPSS Statistics 22.0 (SPSS, Chicago, IL, United States).

\section{Results}

\section{Patients' characteristics}

Our study was based on data obtained from 92 cervical cancer patients between January 2017 and October 2019. The clinicopathological characteristics of 92 cervical cancer patients are shown in Table 1. Initial symptom age was categorized according to three roughly equal strata: $25-39$ years (18 patients, $20.5 \%$ ), and $40-75$ years (70 patients, 79.5\%). Regarding the FIGO stage (2018), stage I was the most common with 58 cases $(63.0 \%)$, and 24 cases were stage II (26.1\%), and 10 cases were stage III and stage IV (10.9\%). The pathological classification was identified as follows: squamous cell carcinoma (63 cases, 68.5\%), adenocarcinoma (15 cases, 16.3\%), and others (14 cases, $15.2 \%)$. The mean age of cervical cancer patients was 49.6 years (range: $25-75$ years), and the mean body mass index (BMI) was $23.1 \mathrm{~kg} / \mathrm{m} 2$ (range: $17.2-35.4 \mathrm{~kg} / \mathrm{m} 2$ ). The mean follow-up period was 14 months (range: 2-31 months). The mean number of lymph nodes dissected was 58 (range: 16-105) during surgery. After the operation, 65 patients $(70.6 \%)$ received adjuvant radiotherapy or chemotherapy, whereas no further treatment was initiated in 27 patients (29.4\%).

\section{Prognostic significance of factors}

By generating Kaplan-Meier curves of progression-free survival (PFS), we found that the initial symptom age 25-39 group had significantly worse PFS $(P=0.020)$ compared to the initial symptom age 40-75 group (Fig. 1 a). Consistent with common sense, our results showed that the stage II, stage III and stage IV group had significantly worse PFS $(P=0.015)$ compared to the stage I (Fig. 1 b). Moreover, the retrieved lymph nodes count 31-85 group showed significantly better PFS $(P=0.002)$ compared to the retrieved lymph nodes count $16-30$ or 85-105 groups (Fig. $1 \mathrm{c}$ ). And the using uterine manipulator group also showed significantly better PFS $(P<0.001)$ compared to the group without uterine manipulator (Fig. 1 d). As presented in Fig. S1, Kaplan-Meier curves of progression-free survival (PFS) suggested that BMI, post-operative bladder catheterization period, human papillomavirus (HPV), pathological classification, pathological differentiation degree, adjuvant radiotherapy or chemotherapy, obstetrical history, Ki-67, estrogen receptor (ER), progesterone receptor (PR), and P53 were not significant predictors for PFS $(P>0.05$ for all $)$ in cervical cancer patients.

\section{Correlation between post-operative bladder catheterization period and potential prognostic factors in cervical cancer}

Bladder dysfunction is one of the most usual long-term side effects and complications after radical hysterectomy 
Table 1 Clinicopathological characteristics of the 92 cervical cancer patients included in this study

\begin{tabular}{|c|c|c|c|}
\hline \multirow[t]{2}{*}{ Characteristic } & \multirow[t]{2}{*}{ Patients } & \multicolumn{2}{|c|}{ Disease Progression $^{\#}$} \\
\hline & & Yes & No \\
\hline \multicolumn{4}{|l|}{ Pathological classification } \\
\hline Squamous cell carcinoma & $63(68.5 \%)$ & 4 & 59 \\
\hline Adenocarcinoma & $15(16.3 \%)$ & 1 & 14 \\
\hline Others & $14(15.2 \%)$ & 1 & 13 \\
\hline \multicolumn{4}{|l|}{ Initial symptom age ${ }^{*}$} \\
\hline $25-39$ & $18(20.5 \%)$ & 3 & 15 \\
\hline $40-75$ & 70 (79.5\%) & 3 & 67 \\
\hline \multicolumn{4}{|l|}{ Days of catheter removal** } \\
\hline $2-7$ & $8(9.2 \%)$ & 2 & 6 \\
\hline$>7$ & 79 (90.8\%) & 3 & 76 \\
\hline \multicolumn{4}{|l|}{ Tumor stage } \\
\hline Stage I & $58(63.0 \%)$ & 1 & 57 \\
\hline Stage ॥ & $24(26.1 \%)$ & 2 & 22 \\
\hline Stagelll + IV & $10(10.9 \%)$ & 3 & 7 \\
\hline \multicolumn{4}{|l|}{ Adjuvant radiotherapy or chemotherapy } \\
\hline Yes & $65(70.6 \%)$ & 6 & 59 \\
\hline No & $27(29.4 \%)$ & 0 & 27 \\
\hline \multicolumn{4}{|l|}{ BMl } \\
\hline Normal & $56(60.9 \%)$ & 6 & 50 \\
\hline Overweight+Obesity+Underweight & $36(39.1 \%)$ & 0 & 36 \\
\hline \multicolumn{4}{|l|}{ Obstetrical history } \\
\hline $0-2$ & $68(73.9 \%)$ & 4 & 64 \\
\hline$>2$ & $24(26.1 \%)$ & 2 & 22 \\
\hline \multicolumn{4}{|l|}{ Retrieved lymph nodes count ${ }^{* * *}$} \\
\hline $16-30$ & $9(10.3 \%)$ & 1 & 8 \\
\hline $31-85$ & $71(81.6 \%)$ & 2 & 69 \\
\hline $86-105$ & $7(8.1 \%)$ & 2 & 5 \\
\hline \multicolumn{4}{|l|}{ Uterine manipulator } \\
\hline Yes & $78(84.8 \%)$ & 5 & 73 \\
\hline No & $14(15.2 \%)$ & 1 & 13 \\
\hline \multicolumn{4}{|l|}{ Pathological differentiation degree ${ }^{* * * *}$} \\
\hline Low differentiation & $19(25.3 \%)$ & 2 & 17 \\
\hline Median differentiation & 49 (65.3\%) & 4 & 45 \\
\hline High differentiation & 7 (9.4\%) & 0 & 7 \\
\hline- & 75 (81.5\%) & 5 & 70 \\
\hline+ & $17(18.5 \%)$ & 1 & 16 \\
\hline
\end{tabular}

${ }^{\circ}$ Missing value $N=4 ;{ }^{* *}$ Missing value $N=5 ;{ }^{* * *}$ Missing value $N=5 ;{ }^{* * * *}$ Missing value $N=17$. ${ }^{*}$ Disease Progression: disease recurrence or death. Abbreviations: $B M I$ body mass index, HPV human papillomavirus

with the incidence of $8-80 \%$. Our study tried to explore the association between the post-operative bladder catheterization period and potential prognostic factors in cervical cancer by Kruskal-Wallis test. As shown in Table 2, stage II, stage III and stage IV group had a significantly longer post-operative bladder catheterization period ( $P=0.026)$ compared to the stage I group. In our study, we also found that the squamous cell carcinoma group had significantly longer post-operative bladder catheterization period $(P=0.023)$ compared to the adenocarcinoma and others group. Particularly, the post-operative bladder catheterization period showed a 


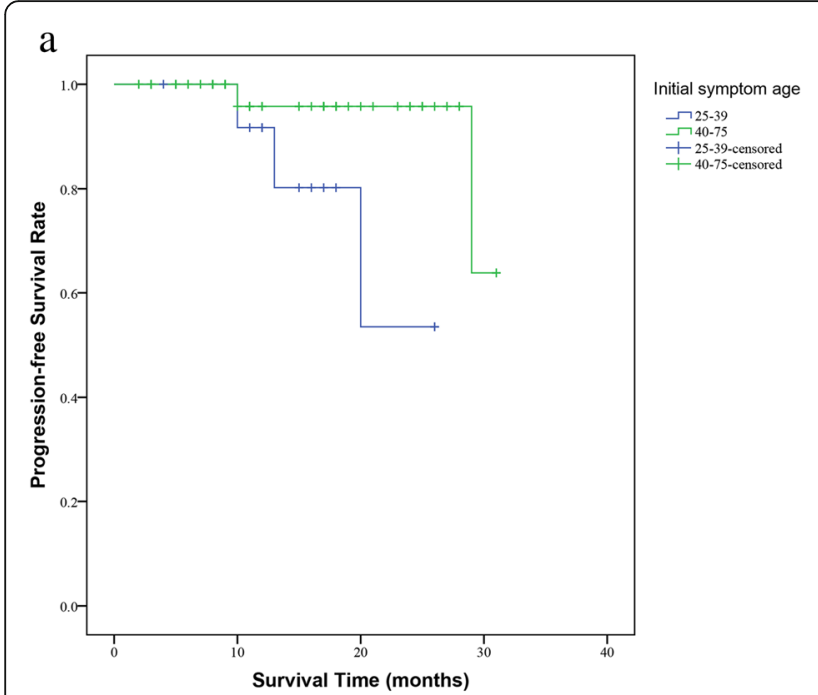

$\mathrm{b}$
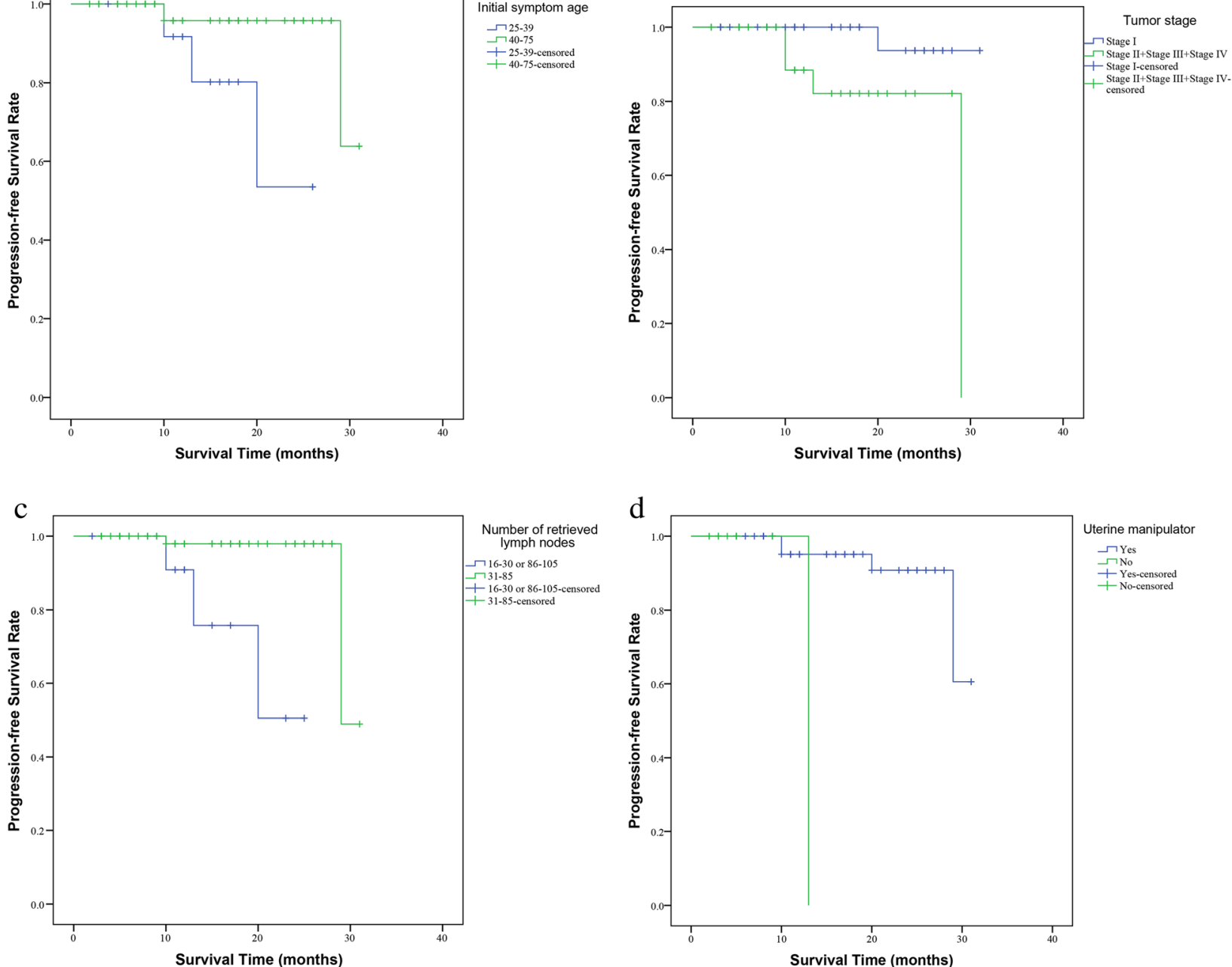

Fig. 1 Kaplan-Meier curves of PFS in patients with cervical cancer. (a): Initial symptom age; (b): Tumor stage; (c): Retrieved lymph nodes count; (d): Uterine manipulator. PFS, progression-free survival

statistical difference between retrieved lymph nodes count 16-30, 31-85, and 86-105 group $(P=0.013)$. However, in the comparison of the characteristics of initial symptom age, BMI, HPV, uterine manipulator, obstetrical history and pathological differentiation degree, no significant difference in post-operative bladder catheterization period was observed between the groups $(P>0.05$ for all).

\section{Combining the FIGO staging system and retrieved lymph nodes count to provide additional stratification}

As shown in Fig. $1 \mathrm{c}$, the number of retrieved lymph nodes count was recommended as an independent prognostic factor. To investigate thoroughly cervical cancer patients with different oncological outcomes and find out more accurate indicators for cervical cancer, this study was implemented to investigate the prognostic roles of combining retrieved lymph nodes count and
FIGO staging system in cervical cancer patients. Herein, cervical cancer patients were stratified by retrieved lymph nodes count and the FIGO staging system as follows: Group1, stage I disease with retrieved lymph nodes count 31-85; Group 2, stage I disease with retrieved lymph nodes count $16-30$ or $86-105$; Group 3, stage II+ III+ IV disease with retrieved lymph nodes count 31-85; Group 4, stage II+ III+ IV disease with retrieved lymph nodes count $16-30$ or $86-105$.

The log-rank test was used to compare the difference in survival between four groups, while the Kaplan-Meier method was used to draw a survival curve. The results revealed that Group 1 had significantly superior survival to the other groups $(P<0.001)$, and the Kaplan-Meier plot also showed that Group 1 had higher survival rates than the other groups (Fig. 2). Receiver operating characteristic (ROC) curve analysis was established to 
Table 2 The association between the post-operative bladder catheterization period and potential prognostic factors

\begin{tabular}{|c|c|c|c|}
\hline Characteristic & $N$ & Mean & $P$-value \\
\hline \multicolumn{4}{|l|}{ Tumor stage } \\
\hline Stage I & 55 & 39.39 & 0.026 \\
\hline Stage II + III + IV & 32 & 51.92 & \\
\hline \multicolumn{4}{|l|}{ Retrieved lymph nodes count } \\
\hline $16-30$ & 9 & 20.44 & 0.013 \\
\hline $31-85$ & 71 & 43.29 & \\
\hline $86-105$ & 7 & 51.71 & \\
\hline \multicolumn{4}{|l|}{ Pathological classification* } \\
\hline Squamous cell carcinoma & 59 & 48.34 & 0.023 \\
\hline Adenocarcinoma & 14 & 36.21 & \\
\hline Others & 13 & 29.38 & \\
\hline \multicolumn{4}{|l|}{ Obstetrical history } \\
\hline $0-2$ & 63 & 43.09 & 0.585 \\
\hline$>2$ & 24 & 46.40 & \\
\hline \multicolumn{4}{|l|}{ Initial symptom age $\mathrm{e}^{* *}$} \\
\hline $25-39$ & 17 & 41.71 & 0.955 \\
\hline $40-75$ & 66 & 42.08 & \\
\hline \multicolumn{4}{|l|}{ BMI } \\
\hline Normal & 53 & 45.92 & 0.374 \\
\hline Overweight+Obesity+Underweight & 34 & 41.00 & \\
\hline \multicolumn{4}{|l|}{ Pathological differentiation degree ${ }^{* * *}$} \\
\hline Low differentiation & 18 & 38.06 & 0.803 \\
\hline Median differentiation & 46 & 35.79 & \\
\hline High differentiation & 7 & 32.07 & \\
\hline \multicolumn{4}{|l|}{ HPV } \\
\hline- & 71 & 44.56 & 0.665 \\
\hline+ & 16 & 41.53 & \\
\hline \multicolumn{4}{|l|}{ Uterine manipulator } \\
\hline Yes & 73 & 43.32 & 0.567 \\
\hline No & 14 & 47.54 & \\
\hline
\end{tabular}

*Missing value $N=1$; ${ }^{* *}$ Missing value $N=4$; ${ }^{* *}$ Missing value $N=16$. Abbreviations: BMI body mass index, HPV human papillomavirus

evaluate the prognostic prediction of the combination of retrieved lymph nodes count (31-85 and 16-30 or 86105) and FIGO staging classification (stage I, II, III and IV). As shown in Fig. 3, the AUC of retrieved lymph nodes count combined with FIGO stage $(\mathrm{AUC}=0.826$, 95\% CI: 0.689-0.962) was better than FIGO staging alone (AUC $=0.748, \quad 95 \%$ CI: 0.561-0.935) in prognostic prediction for cervical cancer patients. These results indicated that the combination of retrieved lymph nodes count (31-85 and $16-30$ or $86-105)$ and FIGO staging classification (stage I, II, III and IV) could act as a potential predictor for prognosis of cervical cancer patients.

\section{Discussion}

Many gynecologic oncologists are dubious about the scope of lymphadenectomy as treating cervical cancer patients with radical hysterectomy. Our study evaluated the effect of the extent of lymphadenectomy on the survival of cervical cancer patients. According to the suggestion of the Gynecologic Oncology Group, the retrieved lymph nodes count that could define an adequate lymphadenectomy was 10 or more [18]. It should maintain a minimum of one from each hemi-para-aortic lymphadenectomy and four lymph nodes from each hemipelvic lymphadenectomy. However, an appropriate number of retrieved lymph nodes for cervical cancer remains questionable. Categorization of cervical cancer patients in published studies is diverse. There is evidence investigated by other researchers demonstrated that divided cervical cancer patients into two groups based on a retrieved lymph nodes cut point of 40 , which the patients could be separated almost equally [9]. Pieterse et al. divided cervical cancer patients into two groups based on the retrieved lymph nodes, a median number of 18 [12]. In our current study, number of retrieved lymph nodes had a statistically significant effect on PFS of cervical cancer patients. Findings of our current study demonstrate that patients with retrieved lymph nodes count 31-85 showed better prognosis than patients with retrieved lymph nodes count 16-30 or 86-105 group. This finding may be conducive to determine surgical scope before surgery for cervical cancer patients. From the results, we can find lymph nodes are not always better dissected as much as possible. We already know the tumors also spread along the lymphatic system. We hypothesis if a higher number of retrieved lymph nodes in a similar dissection area of patients could indicate a more developed lymphatic reflux system, the patients with a more developed lymphatic system would have a higher risk of tumor cell metastasis. Therefore, patients of retrieved lymph nodes count more than 85 in this study had worsening prognosis. This finding may be conducive to determine surgical scope before surgery for cervical cancer patients, and further clinical studies and more data analysis are needed to confirm the details.

Published researches related to the possible significance of initial symptom age of cervical cancer patient as a prognostic factor are contradictory. In several studies, the prognosis is significantly worse in younger cervical cancer patients $[19,20]$. However, in other studies, there is no difference in the survival rates among cervical cancer patients in different age groups [21, 22]. Our results showed that the initial symptom age 25-40 group had significantly worse PFS $(P=0.020)$, and the initial symptom age may be prognostic factor. The relational factors in this result are still unclear. It could not be explained by HPV infection respectively, disease 


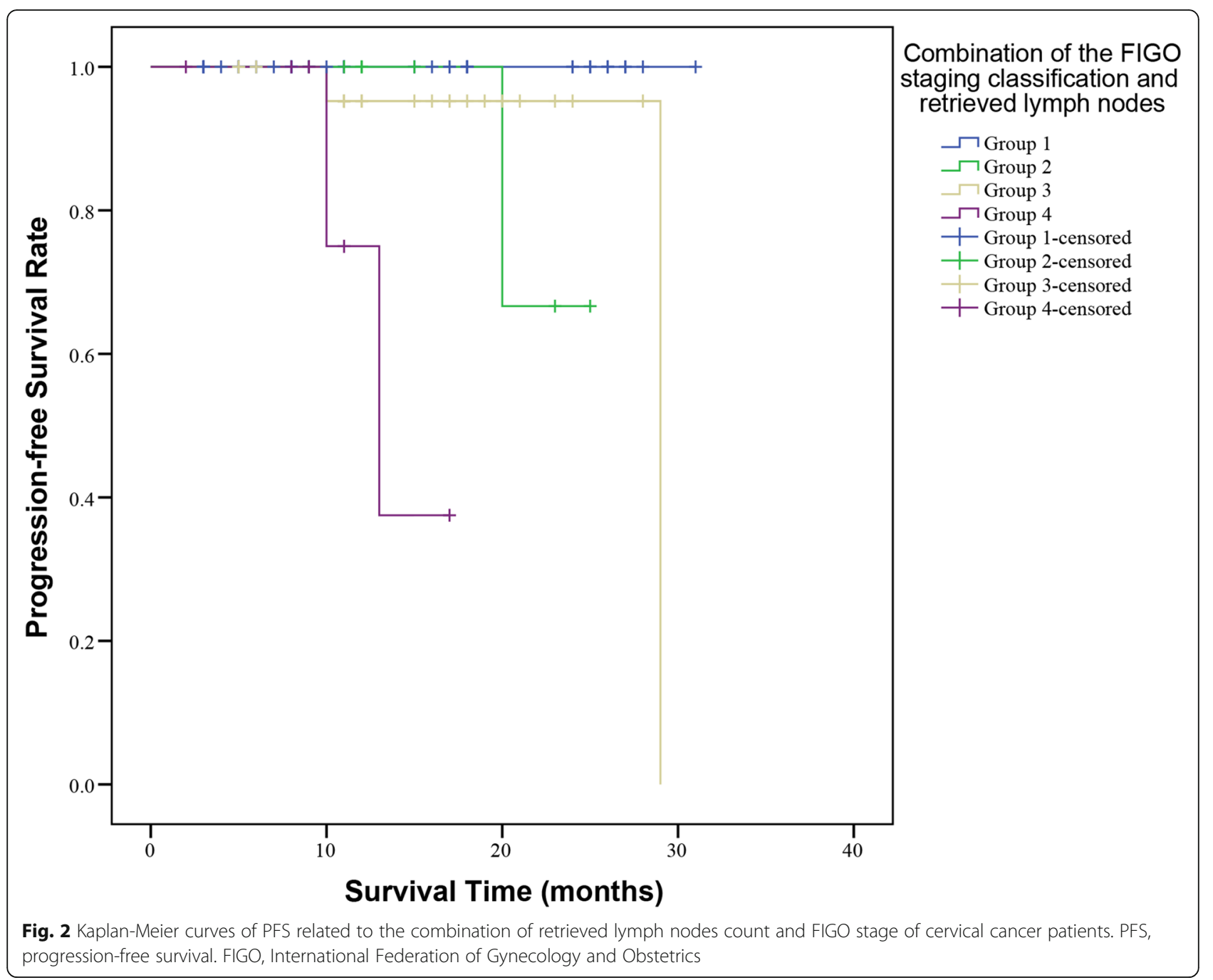

pattern and aggressive characteristics were also potential factor [23]. Agreed with other research, our current study corroborated that stage I cervical cancer patients had significantly better PFS $(P=0.015)$ compared to the stage II, stage III and stage IV cervical cancer patients [24].

While being widely adopted, the using of uterine manipulator in laparoscopic treatment represents a controversial issue. Recent studies have demonstrated that laparoscopic surgery was correlated with worse PFS and shorter overall survival than open abdominal approach among cervical cancer patients $[25,26]$. Uterine manipulator was hypothesized as one of suspected causes responsible for the bad prognosis. However, other studies showed that the use of uterine manipulator in laparoscopic approach does not related to the risk of recurrence and worse PFS [27]. In our current study, the using of uterine manipulator had a statistically significant effect on PFS of cervical cancer patients $(P<0.001)$. Findings of our current study demonstrated that the using of uterine manipulator in laparoscopic treatment showed a better prognosis than patients without uterine manipulator using group. In surgical process of this study, the manipulator was carefully treated and its movements were minimized after placement. The direction should be maintained. The force was controlled as much as possible during the whole surgical process to avoid its violent extrusion of the tumor, which would cause the metastasis of cancer cells. After our observation, if these manipulations above could be achieved, the using of the manipulator could help the surgeon better judging the size of the tumor and surrounding tissue conditions to help patients get better PFS.

A novel discovery of our study was to verify the prognostic values of retrieved lymph nodes count combining with the FIGO staging system, which had never been investigated in cervical cancer before. Our study separated cervical cancer patients into four groups according to the retrieved lymph nodes count and the FIGO staging system. Kaplan-Meier survival curve analysis showed that Group 1(stage I disease with retrieved lymph nodes count 31-85) had higher survival rates than the other 

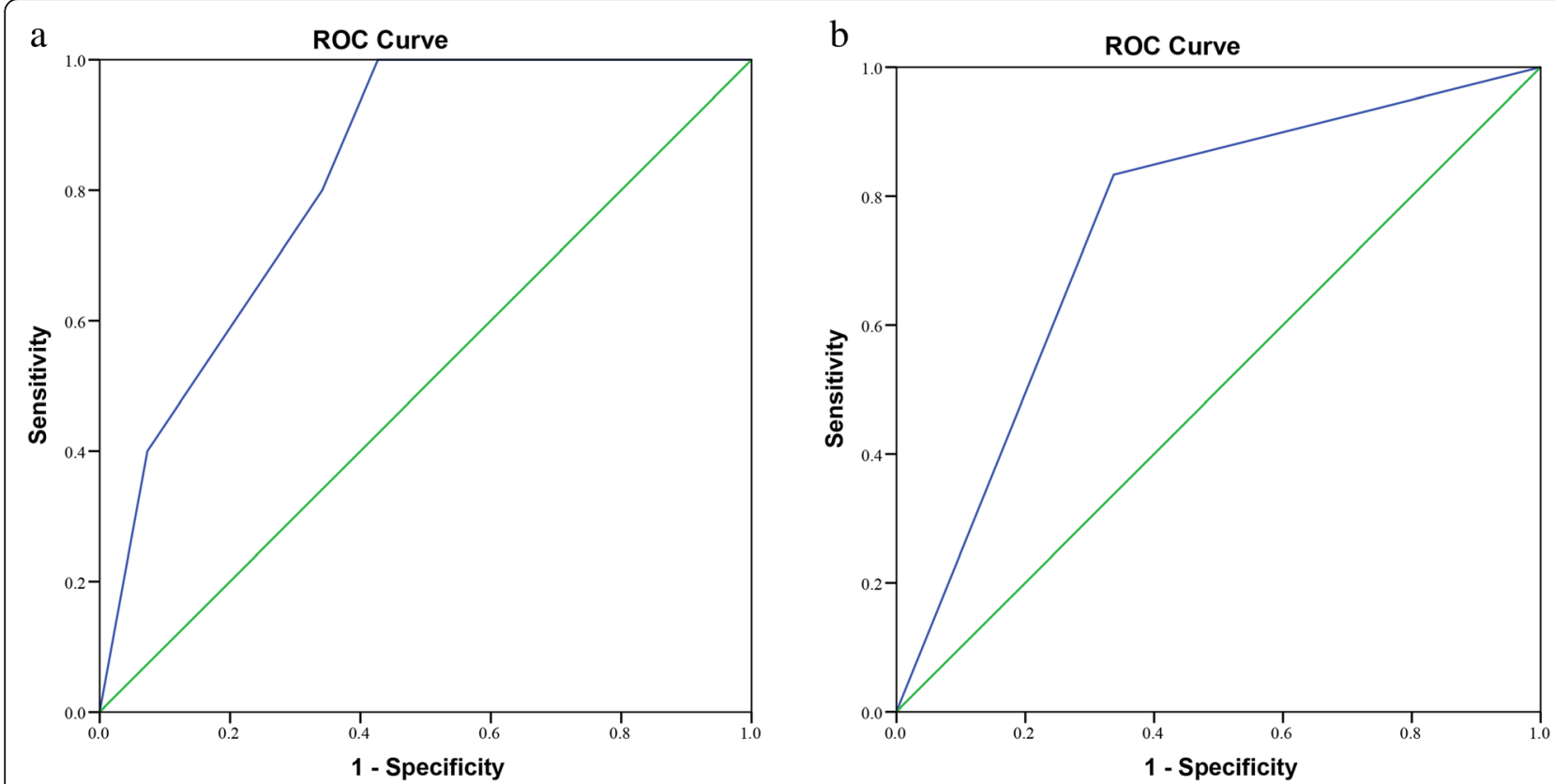

Fig. 3 ROC curves of retrieved lymph nodes count and FIGO staging classification. a: Combination of retrieved lymph nodes count and FIGO staging classification; b: FIGO staging classification. ROC, receiver operating characteristic. FIGO, International Federation of Gynecology and Obstetrics

groups. Receiver operating characteristic (ROC) curve analysis also showed the retrieved lymph nodes count combined with the FIGO stage was better than FIGO staging alone in prognostic prediction for cervical cancer patients. In summary, our study indicates that retrieved lymph nodes count combined with the FIGO stage could be a potential prognostic factor for cervical cancer patients. The combining of retrieved lymph nodes count and FIGO staging system prognostic system would provide added risk stratification, so cervical cancer patients with predicted worse prognosis should receive more aggressive therapeutic regimens.

To date, there is no reported evidence that the postoperative bladder catheterization period has a direct impact on survival. Intraoperative injury of pelvic floor nerve, ureter and bladder were acknowledged factors that affected the days of catheter removal. The time of catheter removal is related to the scope of the operation. Retrieved lymph nodes count reflects the range of surgical dissection, which was found as prognostic factor in this study. Therefore, this study analyzes correlations between potential prognostic factor and days of catheter removal. As shown in results, in the comparison of tumor stage, retrieved lymph nodes count, and pathological type, significant difference in post-operative bladder catheterization period was observed between the groups $(P<0.05$ for all). Based on these results, we raised the possibility that the post-operative bladder catheterization period could be potential indirect acting factor for prognosis of cervical cancer patients. Although it is difficult to demonstrate this hypothesis at present study, further relevant research and verification in the future were expected.

There is no crucial evidence regarding the impact of the pathological differentiation degree of cervical cancer on PFS. Some researches indicate that poorlydifferentiated squamous cell cervical carcinoma has a worse prognosis [28], whereas other researches did not identify $[29,30]$. Another controversial prognostic factor in patients with cervical cancer is the pathological classification [31]. While some studies reported that patients with uterine cervix adenocarcinoma have unfavorable outcomes [32, 33], others identified no significant differences between cervical adenocarcinoma and squamous cell carcinoma [24, 34]. In our current study, it was failed to show pathological differentiation degree and pathological classification were independent prognostic factors. Moreover, our study included four cervical patients with lymphatic obstruction. We speculated that it might connect with retrieved lymph nodes, the scope of lymphadenectomy, and the surgical method. Because the number of samples was limited, our research had some limits. A larger number of cervical cancer samples need to be collected and more studies are also needed to corroborate our speculation.

The standard treatment for recurrence cervical cancer is controversial. There are three diverse treatment choices for local recurrence or persistent cancer: systemic 
therapy or best supportive care or pelvic exenteration [17]. Several studies have shown that diverse surgical approach used in recurrence or persistent cancer could influence the prognosis of cervical cancers patients. Vaginectomy appears to a salvage treatment, for instance, exenteration or radiotherapy with a bottommost impact on quality of life in appropriately high-risk patients [35]. Lateral endopelvic resection (LEPR) was defined as en bloc resection of a pelvic tumor with pelvic side wall (PSW) structures including sidewall muscle, or bone, or major vascular structure, or major nerve to attain negative pathologic margins [36]. It is a curative potential therapeutic option for persistent or recurrent malignancy infiltrating the PSW, especially if chemoradiation failed, associated with prolonged survival [37].

Our current study has several limitations. Firstly, as a retrospective study, the bias could not be neglected. Although 92 cervical cancer patients were included in this study, it is possible that the results might be affected by some confounding factors. Secondly, our study was built on information from a single healthcare institution of a finite geographic region. Therefore, further confirmatory studies at other institutions should be carried out in the future. Finally, the study is still being followed-up and we will continue to follow up the patients in this study until death.

\section{Conclusions}

In this study, we assessed the independent prognostic value of clinical and potential prognostic factors in progression-free survival (PFS) in cervical cancer. Number of retrieved lymph nodes, initial symptoms age, uterine manipulator, and retrieved lymph nodes count combining with FIGO staging system were identified as potential prognostic factors for cervical cancer patients.

\footnotetext{
Abbreviations

PFS: Progression-free survival; ROC: Receiver operating characteristic; LMICS: Low and middle-income countries; NCCRC: National Central Cancer Registry of China; FIGO: International Federation of Gynecology and Obstetrics; RLN: Retrieved lymph nodes; PALND: Para-aortic lymph node dissection; PLND: Pelvic lymph node dissection; HPV: Human papillomavirus; ER: Estrogen receptor; PR: Progesterone receptor
}

\section{Supplementary Information}

The online version contains supplementary material available at https://doi. org/10.1186/s12885-021-08243-3.

Additional file 1: Figure S1. Kaplan-Meier curves of PFS in patients with cervical cancer. (a): Number of births; (b): Adjuvant radiotherapy or chemotherapy; (c): P53; (d): Ki-67; (e): ER; (f): PR; (g): BMl; (h): HPV; (i): Pathological type; (j): Pathological differentiation degree. PFS, progression-free survival.

\section{Authors' contributions}

HHC; Data collection, Manuscript editing, and Protocol. WYM; Data analysis, Manuscript writing and editing. RZL; Project development, Manuscript writing and editing. QYW; Data collection and Protocol. YWW; Project development and Data management. HDP, PYY, QBW and LL; Project development. XJY, MK, EL-HL; Project development and Manuscript editing. All authors read and approved the final manuscript.

\section{Authors' information (optional)}

Not applicable.

\section{Funding}

This work was funded by Administration of Traditional Chinese Medicine of Guangdong Province, China.(Project on:20201325), The Science and Technology Development Fund, Macau SAR (File no: 0096/2018/A3, 0003/ 2019/AKP, 0055/2020/A) and the NSFC overseas and Hong Kong and Macao Scholars Cooperative Research Fund Project (Project no: 81828013). The funding bodies played no role in in the design of the study, data collection, data analysis, data interpretation of data and in writing the manuscript.

\section{Availability of data and materials}

The datasets used and/or analyzed during the current study available from the corresponding author on reasonable request.

\section{Declarations}

\section{Ethics approval and consent to participate}

This study was approved by the institutional ethics committee of Zhuhai Hospital of Traditional Chinese and Western Medicine (Ethic code: 20190412001). All procedures performed in studies involving human participants were in accordance with the ethical standards of the institutional and national research committee, and the consent for participate was approved by the ethics committee.

\section{Consent for publication}

Not applicable.

\section{Competing interests}

The authors declare that they have no competing interests.

Received: 22 September 2020 Accepted: 23 April 2021

Published online: 10 May 2021

\section{References}

1. Bhatla N, Aoki D, Sharma DN, Sankaranarayanan R. Cancer of the cervix uteri. Int J Gynaecol Obstet. 2018;143(Suppl 2):22-36. https://doi.org/10.1 002/ijgo.12611.

2. Bray F, Jemal A, Torre LA, Forman D, Vineis P. Long-term Realism and Costeffectiveness: Primary Prevention in Combatting Cancer and Associated Inequalities Worldwide. J Natl Cancer Inst. 2015;107(12):djv273.

3. Chen W, Zheng R, Baade PD, Zhang S, Zeng H, Bray F, et al. Cancer statistics in China, 2015. CA Cancer J Clin. 2016;66(2):115-32. https://doi.org/10.3322/ caac. 21338.

4. Siegel RL, Miller KD, Jemal A. Cancer statistics, 2019. CA Cancer J Clin. 2019; 69(1):7-34. https://doi.org/10.3322/caac.21551.

5. Plummer M, de Martel C, Vignat J, Ferlay J, Bray F, Franceschi S. Global burden of cancers attributable to infections in 2012: a synthetic analysis. Lancet Glob Health. 2016;4(9):e609-16. https://doi.org/10.1016/S2214-109X(1 6)30143-7.

6. Hu Z, Ma D. The precision prevention and therapy of HPV-related cervical cancer: new concepts and clinical implications. Cancer Med. 2018;7(10): 5217-36. https://doi.org/10.1002/cam4.1501.

7. Zheng RR, Huang XX, Jin C, Zhuang XX, Ye LC, Zheng FY, et al. Preoperative platelet count improves the prognostic prediction of the FIGO staging system for operable cervical cancer patients. Clin Chim Acta. 2017;473:198203. https://doi.org/10.1016/j.cca.2016.11.008.

8. Bipat S, Glas AS, van der Velden J, Zwinderman AH, Bossuyt PM, Stoker J. Computed tomography and magnetic resonance imaging in staging of uterine cervical carcinoma: a systematic review. Gynecol Oncol. 2003;91(1): 59-66. https://doi.org/10.1016/S0090-8258(03)00409-8. 
9. Lim S, Cho K, Lee S, Lee K, Shin J, Chung D, et al. Effect of number of retrieved lymph nodes on prognosis in FIGO stage IB-IIA cervical cancer patients treated with primary radical surgery. J Obstet Gynaecol Res. 2017; 43(1):211-9. https://doi.org/10.1111/jog.13171.

10. Girardi F, Pickel H, Winter R. Pelvic and parametrial lymph nodes in the quality control of the surgical treatment of cervical cancer. Gynecol Oncol. 1993:50(3):330-3. https://doi.org/10.1006/gyno.1993.1220.

11. Panici PB, Scambia G, Baiocchi G, Matonti G, Capelli A, Mancuso S. Anatomical study of Para-aortic and pelvic lymph nodes in gynecologic malignancies. Obstet Gynecol. 1992;79(4):498-502.

12. Pieterse QD, Kenter GG, Gaarenstroom KN, Peters AA, Willems SM, Fleuren $\mathrm{GJ}$, et al. The number of pelvic lymph nodes in the quality control and prognosis of radical hysterectomy for the treatment of cervical cancer. Eur J Surg Oncol. 2007;33(2):216-21. https://doi.org/10.1016/j.ejso.2006.09.037.

13. Frost JA, Webster KE, Bryant A, Morrison J. Lymphadenectomy for the management of endometrial cancer. Cochrane Database Syst Rev. 2017;10: Cd007585.

14. Zullo MA, Manci N, Angioli R, Muzii L, Panici PB. Vesical dysfunctions after radical hysterectomy for cervical cancer: a critical review. Crit Rev Oncol Hematol. 2003;48(3):287-93. https://doi.org/10.1016/51040-8428(03)00125-2.

15. Abu-Rustum NR, Alektiar K, lasonos A, Lev G, Sonoda Y, Aghajanian C, et al. The incidence of symptomatic lower-extremity lymphedema following treatment of uterine corpus malignancies: a 12-year experience at Memorial Sloan-Kettering Cancer Center. Gynecol Oncol. 2006;103(2):714-8. https:// doi.org/10.1016/j.ygyno.2006.03.055.

16. Zhang L, Huang H, Zhang L, Hou T, Wu S, Huang Q, et al. URG4 overexpression is correlated with cervical cancer progression and poor prognosis in patients with early-stage cervical cancer. BMC Cancer. 2014; 14(1):885. https://doi.org/10.1186/1471-2407-14-885.

17. Abu-Rustum NR, Yashar CM, Bean S, Bradley K, Campos SM, Chon HS, et al. NCCN guidelines insights: cervical Cancer, version 1.2020. J Natl Compr Cancer Netw. 2020;18(6):660-6. https://doi.org/10.6004/jnccn.2020.0027.

18. Scott Miller D. Advanced endometrial cancer: is lymphadenectomy necessary or sufficient? Gynecol Oncol. 2006;101(2):191-3. https://doi.org/1 0.1016/j.ygyno.2006.03.027.

19. Dattoli MJ, Gretz HF 3rd, Beller U, Lerch IA, Demopoulos Rl, Beckman EM, et al. Analysis of multiple prognostic factors in patients with stage IB cervical cancer: age as a major determinant. Int J Radiat Oncol Biol Phys. 1989;17(1):41-7. https://doi.org/10.1016/0360-3016(89)90368-4.

20. Stuart GC, Robertson DI, Fedorkow DM, Duggan MA, Nation JG. Recurrent and persistent squamous cell cervical carcinoma in women under age 35 . Gynecol Oncol. 1988;30(2):163-72. https://doi.org/10.1016/0090-82 58(88)90020-0.

21. Meanwell CA, Kelly KA, Wilson S, Roginski C, Woodman C, Griffiths R, et al. Young age as a prognostic factor in cervical cancer: analysis of population based data from 10,022 cases. Br Med J (Clin Res Ed). 1988;296(6619):38691. https://doi.org/10.1136/bmj.296.6619.386.

22. Austin JP, Degefu S, Torres J, Bush DJ, O'Quinn AG, Ozmen N, et al. Cervical carcinoma in women less than 35 years of age. South Med J. 1994;87(3): 375-9. https://doi.org/10.1097/00007611-199403000-00015.

23. Lau HY, Juang CM, Chen YJ, Twu NF, Yen MS, Chao KC. Aggressive characteristics of cervical cancer in young women in Taiwan. Int I Gynaecol Obstet. 2009;107(3):220-3. https://doi.org/10.1016/j.jjgo.2009.07.029.

24. Alfsen GC, Kristensen GB, Skovlund E, Pettersen EO, Abeler VM. Histologic subtype has minor importance for overall survival in patients with adenocarcinoma of the uterine cervix: a population-based study of prognostic factors in 505 patients with nonsquamous cell carcinomas of the cervix. Cancer. 2001;92(9):2471-83. https://doi.org/10.1002/1097-0142(200111 01)92:9<2471::AID-CNCR1597>3.0.CO;2-K.

25. Melamed A, Margul DJ, Chen L, Keating NL, Del Carmen MG, Yang J, et al. Survival after minimally invasive radical hysterectomy for early-stage cervical Cancer. N Engl J Med. 2018;379(20):1905-14. https://doi.org/10.1056/ NEJMoa1804923.

26. Ramirez PT, Frumovitz M, Pareja R, Lopez A, Vieira M, Ribeiro R, et al. Minimally invasive versus abdominal radical hysterectomy for cervical Cancer. N Engl J Med. 2018;379(20):1895-904. https://doi.org/10.1056/ NEJMoa1806395.

27. Yuan P, Liu Z, Qi J, Yang X, Hu T, Tan H. Laparoscopic radical hysterectomy with enclosed Colpotomy and without the use of uterine manipulator for early-stage cervical Cancer. J Minim Invasive Gynecol. 2019;26(6):1193-8. https://doi.org/10.1016/j.jmig.2019.01.016.
28. Landoni F, Maneo A, Cormio G, Perego P, Milani R, Caruso O, et al. Class II versus class III radical hysterectomy in stage IB-IIA cervical cancer: a prospective randomized study. Gynecol Oncol. 2001;80(1):3-12. https://doi. org/10.1006/gyno.2000.6010

29. van Nagell JR, Donaldson ES Jr, Parker JC, van Dyke AH, Wood EG. The prognostic significance of pelvic lymph node morphology in carcinoma of the uterine cervix. Cancer. 1977;39(6):2624-32. https://doi.org/10.1002/10970142(197706)39:6<2624::AID-CNCR2820390648>3.0.CO;2-F.

30. Zaino RJ, Ward S, Delgado G, Bundy B, Gore H, Fetter G, et al. Histopathologic predictors of the behavior of surgically treated stage IB squamous cell carcinoma of the cervix. A Gynecologic Oncology Group study. Cancer. 1992;69(7):1750-8. https://doi.org/10.1002/1097-0142(199204 01)69:7<1750::aid-cncr2820690717>3.0.co;2-s.

31. Winer I, Alvarado-Cabrero I, Hassan O, Ahmed QF, Alosh B, Bandyopadhyay $S$, et al. The prognostic significance of histologic type in early stage cervical cancer - a multi-institutional study. Gynecol Oncol. 2015;137(3):474-8. https://doi.org/10.1016/j.ygyno.2015.02.005.

32. Davy ML, Dodd TJ, Luke CG, Roder DM. Cervical cancer: effect of glandular cell type on prognosis, treatment, and survival. Obstet Gynecol. 2003;101(1): 38-45. https://doi.org/10.1016/s0029-7844(02)02275-5.

33. Chen RJ, Lin YH, Chen CA, Huang SC, Chow SN, Hsieh CY. Influence of histologic type and age on survival rates for invasive cervical carcinoma in Taiwan. Gynecol Oncol. 1999;73(2):184-90. https://doi.org/10.1006/gyno.1 999.5364.

34. Lee KB, Lee JM, Park CY, Lee KB, Cho HY, Ha SY. What is the difference between squamous cell carcinoma and adenocarcinoma of the cervix? A matched case-control study. Int J Gynecol Cancer. 2006;16(4):1569-73. https://doi.org/10.1111/j.1525-1438.2006.00628.x.

35. Vizzielli G, Tortorella L, Conte C, Chiantera V, Gallotta V, Foschi N, Arcieri M, Ferrandina G, Fagotti A, Zattoni $F$ et al: Is a Vaginectomy enough or is a pelvic Exenteration always required for surgical treatment of recurrent cervical Cancer? A Propensity-Matched Study. Ann Surg Oncol 2020.

36. Vizzielli G, Naik R, Dostalek L, Bizzarri N, Kucukmetin A, Tinelli G, et al. Laterally extended pelvic resection for Gynaecological malignancies: a multicentric experience with out-of-the-box surgery. Ann Surg Oncol. 2019; 26(2):523-30. https://doi.org/10.1245/s10434-018-07088-8.

37. Vizzielli G, Chiantera V, Tinelli G, Fagotti A, Gallotta V, Di Giorgio A, et al. Out-of-the-box pelvic surgery including iliopsoas resection for recurrent gynecological malignancies: does that make sense? A single-institution case-series. Eur J Surg Oncol. 2017;43(4):710-6. https://doi.org/10.1016/j. ejso.2016.10.028

\section{Publisher's Note}

Springer Nature remains neutral with regard to jurisdictional claims in published maps and institutional affiliations.

Ready to submit your research? Choose BMC and benefit from:

- fast, convenient online submission

- thorough peer review by experienced researchers in your field

- rapid publication on acceptance

- support for research data, including large and complex data types

- gold Open Access which fosters wider collaboration and increased citations

- maximum visibility for your research: over $100 \mathrm{M}$ website views per year

At BMC, research is always in progress.

Learn more biomedcentral.com/submissions 